\title{
Evasive Higgs Maneuvers at the LHC
}

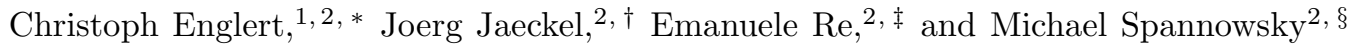 \\ ${ }^{1}$ Institut für Theoretische Physik, Universität Heidelberg, 69120 Heidelberg, Germany \\ ${ }^{2}$ Institute for Particle Physics Phenomenology, Department of Physics, \\ Durham University, DH1 3LE, United Kingdom
}

\begin{abstract}
Non-standard decays of the Higgs boson produced at the Large Hadron Collider can lead to signatures which can easily be missed due to non-adapted trigger or search strategies. Keeping electroweak symmetry breaking Standard Model-like we classify the phenomenology of an evasive Higgs boson into three categories and discuss how they can be described in an effective field theory. We comment on how one can improve the search strategies to also detect such an evasive Higgs.
\end{abstract}

\section{INTRODUCTION}

One of the main goals of the Large Hadron Collider (LHC) is the discovery of the Higgs boson responsible for electroweak symmetry breaking [1]. With the LHC having provided about $5 \mathrm{fb}^{-1}$ of data, both ATLAS and CMS have presented strong constraints on a Standard Model (SM) Higgs boson. If the Higgs is SM-like it is disfavored at the $95 \%$ confidence level in the mass range $131 \mathrm{GeV}(127 \mathrm{GeV})$ to $453 \mathrm{GeV}(600 \mathrm{GeV})$ by ATLAS (CMS) [2, 3], while the LEP2 bound [4] of $114.4 \mathrm{GeV}$ is pushed to $115.5 \mathrm{GeV}(115 \mathrm{GeV})$. With the rapidly growing data sample we can expect to have full coverage of the SM Higgs mass at the $95 \%$ confidence level all the way down to $115 \mathrm{GeV}$ at the LHC for an integrated luminosity of $6 \mathrm{fb}^{-1}[\underline{5}$ ].

The $\mathcal{O}(3 \sigma)$ excesses measured by both Atlas [2, 6] and CMS [3, 7] in the $h \rightarrow \gamma \gamma$ channel might be the first glimpse of a light Higgs (at a considerably larger than expected production cross section) which would be in perfect agreement with all we expect from electroweak precision constraints and measurements performed at LEP and at the Tevatron [9]. However, this might also be just a fluctuation and the Higgs may yet again escape.

What are the implications of these first couple of inverse femtobarns of data for more involved (and better motivated) scenarios of symmetry breaking? ATLAS and CMs have also presented stringent bounds on new physics such as, e.g., the minimal supersymmetric extension of the SM and the SM with the addition of fourth generation. And we expect more model-specific analyses to appear soon, when more data becomes available.

Both ATLAS and CMS provide strong constraints on SM-like Higgs production, even if it is suppressed compared to the SM. These constraints are expressed as limits on $\sigma / \sigma_{\mathrm{SM}}$ and in some mass ranges $\sigma / \sigma_{\mathrm{SM}} \simeq 0.2$ is already excluded at the $95 \%$ confidence level [2, 3, 6 , 8$]$. If a Higgs is to exist in this energy range it has to some-

\footnotetext{
*Electronic address: christoph.englert@durham.ac.uk

${ }^{\dagger}$ Electronic address: joerg.jaeckel@durham.ac.uk

‡Electronic address: emanuele.re@durham.ac.uk

$\S$ Electronic address: michael.spannowsky@durham.ac.uk
}

how "hide" from the Standard searches.

The relevant features for a Higgs search are the production rate and the decay signatures in the detector. Therefore one option to hide the Higgs is to try and significantly reduce the production of the Higgs. Alternatively, the Higgs could dominantly decay into particles which are difficult to detect or difficult to disentangle from the background.

Constraints on our ability to reduce the production cross section arise from the fact that Higgs production rates in the SM are bound to the physical Higgs being the unitarizing degree of freedom in longitudinal gauge boson scattering $V_{L} V_{L} \rightarrow V_{L} V_{L}\left(V=W^{ \pm}, Z\right)$ and in massive $q \bar{q} \rightarrow V_{L} V_{L}$ amplitudes*. In the SM this fixes the partial decay widths and the production cross section for associated Higgs production and weak boson fusion. Essentially this limits the total Higgs production cross section from below (interference effects are typically small [12, 13]). Gluon fusion, $g g \rightarrow h$, is sensitive to the propagating heavy fermionic degrees of freedom and the production rate is again fixed as a function of the Higgs-fermion couplings. Any new physics extension or modification of the Higgs sector has to reproduce these unitarity restoring features to leave a theoretically sound and predictive theory in the LHC-accessible energy range of $\lesssim 3 \mathrm{TeV}$ in the weak boson fusion channel [14], which directly accesses longitudinal gauge boson scattering. In extended Higgs sectors this is typically achieved by linear mixing of the various scalar fields $h_{i}$ such that for energies much larger than $m_{h_{i}}$ the coherent sum of the $h_{i}$ exchange diagrams reproduces the SM Higgs contribution. This is the case for e.g. the two Higgs doublet model, the next to minimal supersymmetric Standard Model [15], composite Higgs models [16] or the Higgs portal scenarios of Refs. [17, 18]. As a result the Higgs production rates (or more precisely the production rate of the light SM-like Higgs) can decrease with a characteristic mixing angle. This can also protect precision electroweak observables such as $S, T, U$ [19] from sizable corrections

\footnotetext{
*Unitarization of $V_{L} V_{L} \rightarrow V_{L} V_{L}$ is a direct consequence of spontaneous symmetry breaking [10], while unitarity in $q \bar{q} \rightarrow V_{L} V_{L}$ relates the fermion and gauge sectors and is less obvious, see e.g. Ref. [11].
} 
from high mass scales. However, unless we appeal to finetuning, accounting for both electroweak precision data and unitarity at the same time typically amounts to a light SM-like Higgs boson with significant production cross section 20, 21]. Finally, smaller Higgs production cross sections can also be obtained from anomalous Higgs couplings [22].

The most minimal assumption is that electroweak symmetry breaking is caused by a single $S U(2)$ doublet Higgs. In this paper we will use this assumption and therefore we will focus on invisible decays and modified signatures. In the following we investigate how a simple extension of the Higgs sector can lead to a "hidden" Higgs phenomenology at the LHC. Generically, the recent LHC bounds can be weakened or even avoided this way.

Such Higgs "hide-out" scenarios are due to a combination of dynamics and kinematics, e.g. they occur through modified branching ratios as a consequence of an extended spectrum or modified couplings. There is a plethora of theoretically sound models which do such modifications and hide the Higgs [18, 21, 23 25]. Therefore, a classification on the level of the phenomenological outcome is desirable. In the following we will pursue this approach. Nevertheless, we also provide an interpretation in terms of a simple effective model. Our categorization is of course "non-invertible"; many different models [26] exhibit a similar phenomenology, and we do not try to compile an exhaustive list of model-building realizations of a specific phenomenological outcome. A minimal realization is the coupling of the otherwise SMlike Higgs to a hidden sector via a renormalizable "portal" interaction $^{\dagger} \sim|H|^{2} \mathcal{O}_{\text {hid }}[18]$. Although it is one of the simplest gauge invariant and renormalizable extensions we can come up with to model a Higgs hide-out, it is an example of how to evade the currently existing bounds on SM Higgs and encompasses a huge range of phenomenological characteristics.

Starting from our assumption of an essentially SM-like electroweak symmetry breaking mechanism, our aim is to categorize the variety of Higgs hide-outs in terms of their phenomenological signatures. At the same time we want to provide a simple parametrization in terms of an effective Lagrangian that realizes these features. In section [I] we review and collect the necessary ingredients for the Lagrangian. In Sect. IIA we consider a dominantly invisibly decaying Higgs and study the implications of current LHC data. Then in Sect. IIB we look at a Higgs decaying into long lived particles that could be searched for by displaced vertex searches. Here we provide additional motivation to also search in the outer parts of the detector. Finally the Higgs could also be buried in

${ }^{\dagger}$ There are two more such portals: kinetic mixing with an extra $U(1)$ gauge group (see e.g. Ref. [27] and Ref. 28] for a review of some low energy consequences), and neutrinos mixing with sterile neutrinos (cf. [29]). large SM backgrounds (Sect. IIC). While this cannot be achieved by changing the gluon coupling alone we find that it is possible in scenarios with enhanced couplings to light quarks or when heavy flavor mesons decaying into gluons dominate the Higgs decay chain. We briefly discuss combinations of these scenarios in Sect. IID, We conclude this paper with a summary in section

\section{PHENOMENOLOGICAL HIGGS HIDE-OUTS}

To achieve a situation in which the Higgs phenomenology can be hidden we have to introduce an extension of the SM Higgs sector which preserves $S U(2) \times U(1)$ gauge invariance. We limit ourselves to renormalizable interactions in the Higgs sector. The only choice is the addition of a scalar interacting via the previously mentioned Higgs portal

$$
\mathcal{L}=\mathcal{L}_{\mathrm{SM}}+\eta|H|^{2}|\phi|^{2}+\partial_{\mu} \phi^{\star} \partial^{\mu} \phi-m^{2}|\phi|^{2} .
$$

The field $\phi$ is taken to be a singlet under the SM gauge group. One could have non-trivial representations of $\phi$ under $S U(2)_{L} \times U(1)_{Y}$ in the Higgs sector, which admits more involved dynamics than Eq. (1). If these extra degrees of freedom are phenomenologically hidden, we encounter a situation which still can be meaningfully described by Eq. (11). Throughout, we define the field $H$ to be responsible for electroweak symmetry breaking. $\phi$ can live in the scalar or vectorial representation of the Lorentz group but can in principle also effectively arise from a fermionic condensate of a strongly interacting sector. As an additional simplification one can impose a $U(1)$ or $\mathbb{Z}_{2}$ symmetry which forbids terms $\sim \phi$.

In general there could also be more than one field $\phi$. This can allow for a variety of decay cascades in the hidden sector. In our study we will concentrate on the following simple set of interactions,

$$
\begin{aligned}
\mathcal{L}_{\text {multi }} & =\sum_{i=1}^{n} \partial_{\mu} \phi_{i}^{\star} \partial^{\mu} \phi_{i}-m_{i}^{2} \phi_{i}^{\star} \phi_{i}+\partial_{\mu} \phi_{i}^{\prime \star} \partial^{\mu} \phi_{i}^{\prime}-m_{i}^{\prime 2} \phi_{i}^{\prime \star} \phi_{i}^{\prime} \\
& +\sum_{i=1}^{n-1} \rho_{i} \phi_{i}^{\star} \phi_{i+1} \phi_{i+1}^{\prime}+\text { h.c. }
\end{aligned}
$$

If desired one can choose charges such that the $U(1)$ symmetry is preserved. We take the particles to be ordered in mass, allowing cascade decays. We identify the heaviest state $\phi_{1}$ with $\phi$ in Eq. (1). Also we will typically allow only the last particles $\phi_{n}$ of the decay cascade to decay into SM particles accordingly. If a cascade is considered one has to replace $\phi \rightarrow \phi_{n}$ in Eq. (5) below.

After the Higgs acquires a vacuum expectation value we induce a BSM trilinear coupling of the physical Higgs $h$ to $\phi$

$$
\epsilon h \phi^{\star} \phi \quad \text { with } \quad \epsilon=\eta\langle H\rangle=\eta v / \sqrt{2}
$$


which modifies the Higgs branching ratios for the Higgs decay. In addition the physical mass $m_{\phi}$ is given by

$$
m_{\phi}^{2}=m^{2}-\eta\langle H\rangle^{2}=m^{2}-\eta v^{2} / 2,
$$

which we take to be positive. Note that since $\phi$ does not develop a vacuum expectation value, there is no mixing of the two scalar states. Accordingly cross sections and decay rates are not modified by mixing effects.

If unbroken, the global $U(1)$ symmetry forbids decays of $\phi$ into SM particles. Hence, in order to re-introduce such decays we need $U(1)$ breaking couplings to SM particles. The SM gauge symmetries forbid couplings of SM matter and gauge fields to $\phi$ on the renormalizable level. We will therefore consider the following dimension 5 couplings (which automatically also explicitly break the $U(1)$ symmetry),

$$
\begin{aligned}
\mathcal{L} & \supset \sum_{i} \frac{\lambda_{i j}}{M} \phi \bar{D}_{L, i} H \Psi_{R, j}+\text { h.c. } \\
& +\left[\frac{\kappa_{\gamma}}{M}\left(\phi+\phi^{\star}\right) F^{\mu \nu} F_{\mu \nu}+\frac{\tilde{\kappa}_{\gamma}}{M}\left(\phi-\phi^{\star}\right) F^{\mu \nu} \tilde{F}_{\mu \nu}\right] \\
& +\left[\frac{\kappa_{g}}{M}\left(\phi+\phi^{\star}\right) G^{\mu \nu} G_{\mu \nu}+\frac{\tilde{\kappa}_{g}}{M}\left(\phi-\phi^{\star}\right) G^{\mu \nu} \tilde{G}_{\mu \nu}\right]
\end{aligned}
$$

where $D_{L}$ denotes the left-handed fermion doublet and $\Psi_{R}$ the right handed fermions. In our effective theory approach we can choose suitable $\lambda_{i j}$ to avoid flavor, lepton number and baryon number changing processes [30]. $\tilde{F}, \tilde{G}$ are the dual QED and QCD field strength tensor ${ }^{\ddagger}$. These higher dimensional operators allow $\phi$ to decay back to visible SM matter.

Since we take the Higgs to be responsible for electroweak symmetry breaking the partial decay widths for $h \rightarrow V V$ are fixed. However, we can model modifications of the branching ratios and the total decay width by introducing an additional contribution to the coupling

$$
\mathcal{L}_{g g h}=\chi \frac{\alpha_{s}}{12 \pi v} h G^{\mu \nu} G_{\mu \nu},
$$

(see Ref. 31] for theoretical bounds) or operators of the form

$$
\mathcal{L}_{H H q q}=\frac{\beta_{Q_{L}}}{M^{2}} H^{\dagger} H \bar{Q}_{L} \not D Q_{L} .
$$

Of course one can also add similar couplings for the right handed quarks and leptons.

\section{A. Hidden Higgses}

If the global $U(1)$ symmetry of the $\phi$-field is unbroken or extremely weakly broken, i.e. the couplings given in

\footnotetext{
${ }^{\ddagger}$ If kinematically possible, i.e. for very high Higgs masses, one could also add an additional term for decays to $W^{ \pm}, Z$. This however would not lead to a phenomenologically very different situation.
}

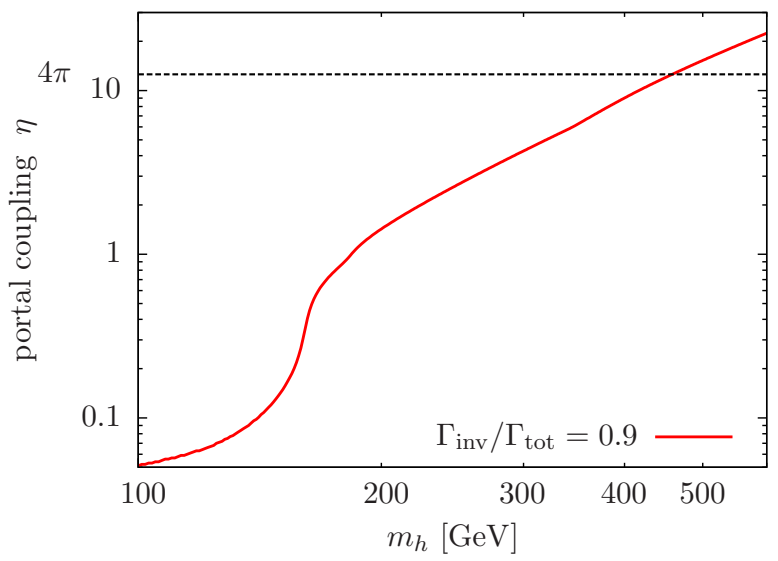

FIG. 1: Required size of the Higgs portal coupling $\eta$ to achieve an invisible branching ratio of $\mathrm{BR}$ (invis) $=90 \%$. We choose $m_{\phi}=10 \mathrm{GeV}$, the result is however rather independent of this choice.

Eq. (5) vanish or are very small, $\phi$ is stable with respect to decays into Standard Model particles. Accordingly, the decay $h \rightarrow \phi^{\star} \phi$ (possible as long as $m_{h} \geq 2 m_{\phi}$ ) is invisible.

Such invisible decays make search strategies based on visible SM particles more difficult. Naively, one can again use that current Higgs searches are already sensitive to cross sections lower than the SM cross section. Using this one can reinterpret the limits on $\sigma_{h} / \sigma_{h}^{\mathrm{SM}}$ as limits on $\sigma_{h} \mathrm{BR}(h \rightarrow \mathrm{SM}) / \sigma_{h}^{\mathrm{SM}}$.

The partial decay width for $h \rightarrow \phi^{\star} \phi$ is given by

$$
\Gamma_{\mathrm{inv}}=\frac{\eta^{2} v^{2}}{16 \pi} \frac{\left[\left(m_{h}^{2}-4 m_{\phi}^{2}\right)\right]^{1 / 2}}{m_{h}^{2}} .
$$

As can be seen from Fig. 1 at low Higgs masses quite moderate values of $\eta$ are sufficient to achieve dominantly invisible decays, allowing to evade search strategies based on SM particles for now. Even when we later on look at modified decays to SM particles this makes it more difficult to hide the Higgs in the high mass region. Above the $V V$-threshold, however, the hidden sector decay width has to compete with a much larger and rapidly growing $\sim m_{h}^{3}$ decay width into SM particles. This requires fairly large values of the Higgs portal coupling $\eta \gtrsim 1$ for $m_{h} \gtrsim 180 \mathrm{GeV}$ and even $\eta \gtrsim 4 \pi$ for $m_{h} \gtrsim 450 \mathrm{GeV}$. The reason for this is as simple as compelling: Unitarization of $V V$ scattering requires a sufficiently large coupling of the Higgs to energetic longitudinal $V$ s resulting also in a large particle decay width of the Higgs into those if the Higgs is heavy. The required large couplings to hide the Higgs into invisible decays are at odds with perturbativity unless they are connected to spontaneous symmetry breaking. Therefore, at large Higgs masses hiding the Higgs into invisible decays is difficult from a model building point of view. For low Higgs masses the Higgs can be easily hidden in invisible decays and alternative strategies [33, 34] based on missing energy $\left(\mathbb{E}_{T}\right)$ searches then become necessary. 


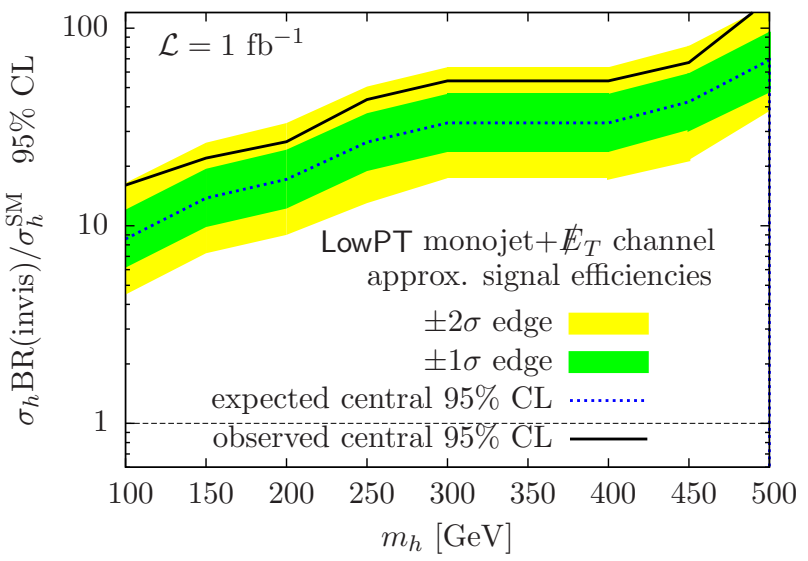

FIG. 2: Expected and observed $(\langle\xi\rangle=0.27) 95 \%$ CL upper limits on the production cross section in multiples of the SM cross section for a monojet $+\mathbb{E}_{T}$ search confronted with an invisibly decaying Higgs.

An important constraint for hidden Higgses comes from direct searches of associated hidden Higgs production at LEP. For SM-like Higgs gauge boson couplings this puts a lower limit of $114.4 \mathrm{GeV}$ on the mass of a dominantly invisibly decaying Higgs [35].

\section{Monojet + missing energy channel}

At the LHC the production of a light Higgs boson is dominated by the gluon fusion process $g g \rightarrow h+X$. Accordingly a search in the monojet plus missing energy channel is promising [36] because backgrounds are comparably small and can be brought under sufficient control [37]. We focus in the following on a center of mass energy of $7 \mathrm{TeV}$, which allows to relate our results to the current LHC run.

We compute the $g g \rightarrow h+X$ signal using an NLO computation matched to a parton shower ${ }^{\S}$ : the inclusive cross section is therefore NLO accurate, the hardest jet (relevant for this study) is described with the full $h+1$ jet matrix element accuracy and further emissions are generated in the shower approximation. We use the Powheg method (as implemented in the Powheg Box program) 39], together with (transverse-momentum ordered) Pythia 6 [40]. We generate events for values of $m_{h}$ between 100 and $500 \mathrm{GeV}$, using the narrow-width approximation.

The Higgs-boson is set stable and excluded from the tracks entering the analysis. On all the other final state particles we apply the "LowPT" selection cuts described in Ref. [37]. Jets are constructed using the anti- $k_{t}$ algo-

\footnotetext{
$\S$ The matching prescription in this channel is subject to an ongoing discussion in the corresponding community, see Refs. [38, 39] for details. Our results do not include any theoretical uncertainties.
}

rithm [41], with $R=0.4$, and we keep only events where

$$
\begin{aligned}
& p_{T}^{j_{1}}>120 \mathrm{GeV},\left|\eta^{j_{1}}\right|<2 \\
& p_{T}^{j_{2}}<30 \mathrm{GeV} \text { if }\left|\eta^{j_{2}}\right|<4.5 \text { (jet-veto) } \\
& \mathbb{E}_{T}>120 \mathrm{GeV} .
\end{aligned}
$$

We obtain $\mathbb{E}_{T}$ as the transverse momentum of the total momentum from all the visible particles, defined to be final state particles with $p_{T}>2 \mathrm{GeV}$ and $|\eta|<4.5$. We also rejected the (rare) events where there is at least one lepton within the cuts defined in Ref. [37].

We extract the background distribution and the data for a luminosity $\mathcal{L}=1 \mathrm{fb}^{-1}$ from Ref. [37].

In Fig. 2 we plot the resulting $95 \%$ confidence level (CL) upper bound on $\sigma_{h} \mathrm{BR}$ (invis) $/ \sigma_{h}^{\mathrm{SM}}$ applying the $\mathrm{CL}_{S}$ method ${ }^{\top}$.

We estimate the average efficiency $\langle\xi\rangle$ that relates the theoretical Higgs cross section prediction for a given mass to the experimentally observable one by performing a Monte Carlo study relating the results of the dominant and signature-wise similar $(Z \rightarrow$ inv) + jets background to the expected numbers quoted in Ref. [37]. For this purpose we produce a $(Z \rightarrow$ inv) + jets event sample using SHerpa [44] and normalize it to the next-to-leading order QCD cross section obtained with MCFM [45] $\left(\sigma_{(Z \rightarrow \bar{\nu} \nu)+j}^{\mathrm{NLO}}\left(p_{T}^{j} \geq 120 \mathrm{GeV},\left|\eta_{j}\right| \leq 2.0\right)=41.3 \mathrm{pb}\right)$. Running the analysis with the above cuts we can estimate $\langle\xi\rangle=0.27$ and we use this significance to rescale our Higgs signal hypotheses in Fig. 2. The observed limits are weaker than the expected limits because of a slight excess of the central data values [37].

The confidence level scales as $\sim \mathcal{L}^{-1 / 2}$. While searches with present luminosities are insensitive to SM-like production cross sections $(\chi \simeq 1$ in Eq. (6) $)$ enhanced production cross sections $(\chi \simeq 3-5)$ occurring in a variety of models are already constrained. In particular for fourth generation models [46 49] where we expect $\chi \simeq 3$, this will very soon become a relevant constraint. With the fast growing data sets these constraints will tighten significantly in the near future.

$$
\text { Two Leptons }+\mathbb{E}_{T} \text { Channel }
$$

A very clean and therefore important search channel for hidden Higgs decays is associated production $p p \rightarrow h Z$ with subsequent decay of the $Z$ to leptons [33, 50]. Note that for associated production, it is much more difficult to obtain increased cross sections since the relevant coupling is fixed by gauge invariancell. For completeness and comparison with the previous section, we

T The $\mathrm{CL}_{S}$ method procedure is fairly standard method in nowadays experimental analysis to present constraints in new physics searches. We refer the read to Refs. [42, 43] for further details.

$\|$ This fact also accounts for the hidden higgs search in the weak boson fusion channel discussed in [51]. For a center of mass en- 


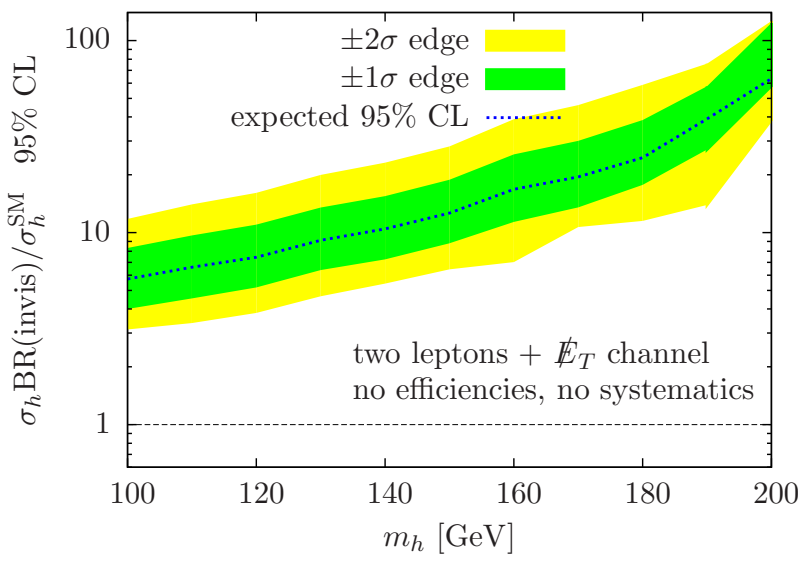

FIG. 3: Expected 95\% CL upper limits on the associated production cross section in multiples of the SM cross section for a two leptons $+\mathbb{E}_{T}$ search confronted with an invisibly decaying Higgs. Superficially this channel looks more sensitive then the monojet search. Note, however, that this plot does not include any efficiencies and is not based on actual data.

nonetheless estimate the performance of the corresponding search. To our knowledge there is no publicly available LHC result of the processes in the phase space region we are interested in. Hence the results of this section are obtained from Monte Carlo, and we include neither background nor signal systematics (this includes a potential mismeasurement of $Z+$ jets, giving rise to a finite $\mathbb{E}_{T}$ by detector effects). Therefore, the sensitivity in this channel is obviously optimistic.

The cross section for associated Higgs production is much smaller than the one for gluon fusion, and shape comparisons of e.g. the $p_{T}^{Z}$ distribution are not possible given $\lesssim 10$ signal events for $\mathcal{L}=1 \mathrm{fb}^{-1}$. Instead we perform a counting experiment for the signal and dominant $Z Z, W W$ and $t \bar{t}$ backgrounds. Again we compute the signal and background cross sections at $\sqrt{s}=7 \mathrm{TeV}$ using MCFM. We require two oppositely charged leptons of identical flavor to combine to the $Z$ mass within a $\pm 10 \mathrm{GeV}$ mass window and $\mathbb{E}_{T} \geq 100 \mathrm{GeV}$.

Our resulting estimate on the upper 95\% CL is shown in Fig. 3. For very low masses the two leptons $+\mathbb{E}_{T}$ channel can be of similar importance as the monojet $+\mathbb{E}_{T}$ search, depending on the signal and background efficiencies. For higher Higgs masses this channel looses sensitivity very quickly due to the small cross section at $7 \mathrm{TeV}$

ergy of $7 \mathrm{TeV}$ weak boson fusion is not an important channel since for typical search cuts and $\mathcal{L} \simeq 1 \mathrm{fb}^{-1}$. We find a reduced cross section [52] $\sigma(7 \mathrm{TeV}) / \sigma(14 \mathrm{TeV}) \simeq 0.2$ compared to $14 \mathrm{TeV}$. As a consequence searches based on small angular separations of the two tagging jets are less sensitive compared to other channels if one also takes into account the systematic uncertainties of the central jet veto and the forward tag jet energy scale uncertainty due to pile-up. Only recently, with the $5 \mathrm{fb}^{-1}$ set CMs has started to overcome these systematic limitations [53]. center of mass energy. Eventually this channel will become again important for large luminosities $\mathcal{O}\left(100 \mathrm{fb}^{-1}\right)$ at $\sqrt{s}=14 \mathrm{TeV}[33]$.

\section{B. Reemerging Higgses}

In some cases the "invisible" decay products of the previous subsection can decay back into SM particles and the hidden Higgs slowly reemerges. In our toy model this is realized when the $U(1)$ violating couplings of Eq. (5) are turned on. At leading order the decay rate of $\phi$ is given by,

$$
\Gamma_{\phi}=\frac{Y^{2}}{4 \pi} \frac{\left(m_{\phi}^{2}-4 m_{f}^{2}\right)^{3 / 2}}{2 m_{\phi}^{2}}
$$

where $Y=\lambda v /(\sqrt{2} M)$ is the effective Yukawa coupling that results from the lagrangian Eq. (5). If the couplings are very small, i.e. the mass scale $M$ is very high, $\phi$ can travel a measurable distance before decaying,

$$
d=\frac{\beta \gamma}{\Gamma_{\phi}}
$$

In this case one may search for (highly) displaced vertices [54] or use adapted trigger strategies [55, 56]. If decays happen inside the tracker this is a fairly clean signature. If the decay length is of the order of meters and above a significant part of the decays will happen in the bigger outer parts of the detector (or even outside the detector). In this case one can gain additional sensitivity by also triggering on events where the decay happens outside of the tracker. If the decay happens outside the detector coverage the same search strategies outlined in Sec. II A apply.

The LHC experiments ATLAS and CMS consist each of four different layers: the inner tracker, the electromagnetic $(\mathrm{E})$ calorimeter, the hadron $(\mathrm{H})$ calorimeter and the

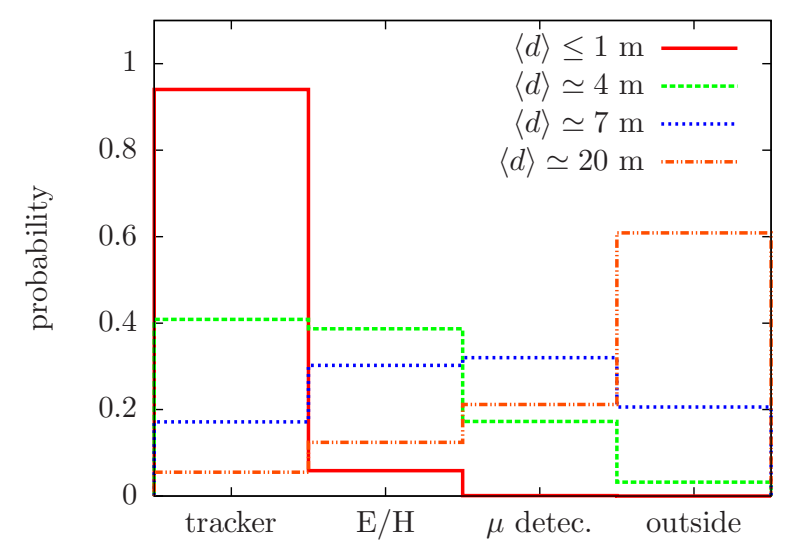

FIG. 4: Probability, based on the AtLAs geometry, for a $\phi$ to decay in the tracker, E/H-calorimeter, muon-calorimeter or outside the detector. We show the results for four different average lab frame decay lengths, respectively. 


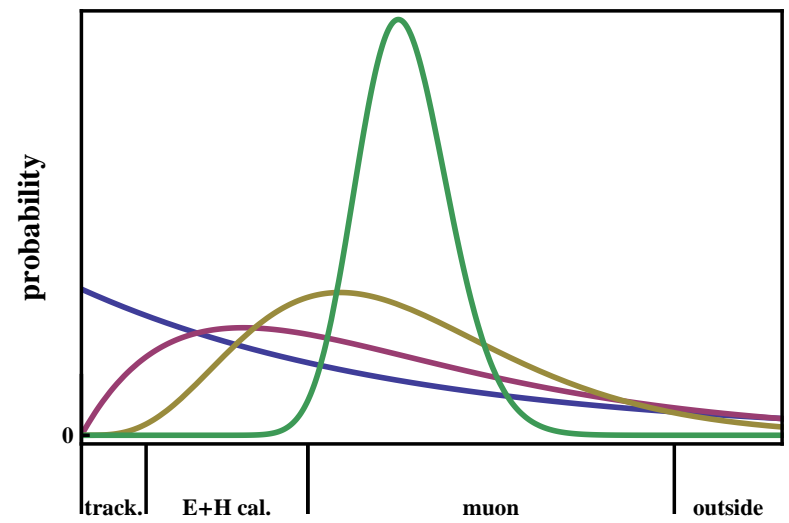

FIG. 5: Probability for the distance between initial Higgs production and final decay back into SM particles for a cascade decay with 1 (blue), 2 (red), 5 (yellow) and 50 (green) intermediate steps.

muon calorimeter. The sensitivity on highly displaced vertices is limited by the radial extension of the experiments. The radial size of the different detector components differs between the two experiments, particularly for the muon calorimeters. We focus in the following analysis optimistically on the geometry of the larger ATLAS experiment.

The muon calorimeter of the ATLAS experiment is located $d \lesssim 11 \mathrm{~m}$ away from the interaction point. The muon detector coverage in pseudorapidity is $|\eta|<$ 2.4. In the muon calorimeter photons and electrons will be stripped in an early layer after the conversion $\phi \rightarrow f \bar{f}$, hence, are likely to be misinterpreted as detector noise [57]. Assuming the Higgs boson decays instantaneously the probability for $\phi$ to decay between distances $d_{1}$ and $d_{2}>d_{1}$ is given by

$$
p\left(d_{1} \leq d \leq d_{2}\right)=\int_{d_{1}}^{d_{2}} \mathrm{~d} x \frac{\Gamma}{\beta \gamma} \exp \left(-\frac{\Gamma x}{\beta \gamma}\right) .
$$

In the lab frame the decay of $h \rightarrow \phi^{\star} \phi$ induces a significant boost factor $\beta$ for $\phi$. We find, that the transverse momentum of the Higgs boson generated from initial state radiation is of minor importance for $\beta$, because the Higgs rarely decays along its direction of motion. Based on Eq. (12) we show the probability of $\phi$ decaying respectively in the tracker, the $\mathrm{E} / \mathrm{H}$-calorimeter, the muon-calorimeter or outside the detector in Fig. 目 for a number of decay lengths. We include the effects of finite detector coverage $|\eta| \leq 2.4$ for a particle cluster with $p_{T} \geq 2 \mathrm{GeV}$ and correct on the longitudinal dimension of the detector.

So far the larger number of decays happening in the outer parts of the detector arise simply from the fact that these parts are bigger. However, if we allow for cascade decays $h \rightarrow \phi_{1} \rightarrow \phi_{2} \rightarrow \ldots \rightarrow \mathrm{SM}$ as described by the Lagrangian Eq. (2) one can achieve that more decays happen close to the average total decay length. For the simple case when subsequent decays generate negligible transverse momentum (i.e. when the mass difference to the sum of the masses of the decay products is small) and the decay lengths are all equal $\lambda / n$ the resulting probability distribution for an $n$-step decay is

$$
P_{n}(x)=\frac{\exp (-n x / \lambda) n(n x)^{n-1} \lambda^{-n}}{(n-1) !} .
$$

This is shown in Fig. [5 In this way one can reduce the number of decays happening in the inner tracker compared to decays occurring in outer parts increasing the need to also check for decays there.

\section{Buried Higgses}

Naively we can also try to hide the Higgs by burying it in the busy hadronic final state at the LHC. In our effective theory approach we can facilitate this by increasing its branching ratio to gluons via the operator of Eq. (6). The modified decay width to gluons then scales like

$$
\Gamma(h \rightarrow g g)=\chi^{2} \Gamma_{\mathrm{SM}}(h \rightarrow g g) .
$$

The same effect increases the higgs production cross section from gluon fusion by the same factor, yielding modified production times branching ratio factors for the individual higgs decay channels $g g \rightarrow h \rightarrow i i^{(\dagger)}$

$$
\begin{aligned}
& i \neq g: \quad \frac{[\sigma \mathrm{BR}]}{[\sigma \mathrm{BR}]_{\mathrm{SM}}}=\frac{\chi^{2}}{\left(\chi^{2}-1\right) \mathrm{BR}_{\mathrm{SM}}(h \rightarrow g g)+1}, \\
& i=g: \quad \frac{[\sigma \mathrm{BR}]}{[\sigma \mathrm{BR}]_{\mathrm{SM}}}=\frac{\chi^{4}}{\left(\chi^{2}-1\right) \mathrm{BR}_{\mathrm{SM}}(h \rightarrow g g)+1} .
\end{aligned}
$$

For the "standard" search channels we have

$$
\frac{[\sigma \mathrm{BR}]}{[\sigma \mathrm{BR}]_{\mathrm{SM}}} \gg 1 \text { for } \chi \gg 1,
$$

since for light Higgs masses we have $\mathrm{BR}_{\mathrm{SM}}(h \rightarrow g g)=$ $\mathcal{O}(1 \%)$. As a result the Higgs is not buried but even more visible in the standard search channels. Note, however, that the increased total width of the Higgs boson will give rise to reduced reconstruction efficiencies in standard search channels**.

One way to bury the Higgs in hadronic final states is to enhance one of the couplings to light quarks, for example using the operator of Eq. (77). This reduces the branching ratios for the standard search signatures without significantly increasing the production cross section, since gluon fusion dominates the production. This might be one of the most difficult channels to uncover.

Alternatively we can again consider decays to $\phi$ s which promptly decay and back into SM quarks or gluons. This

\footnotetext{
** In principle a similar effect can arise if we dramatically increase an invisible decay width. The monojet and other invisible Higgs search strategies should be less affected by this.
} 


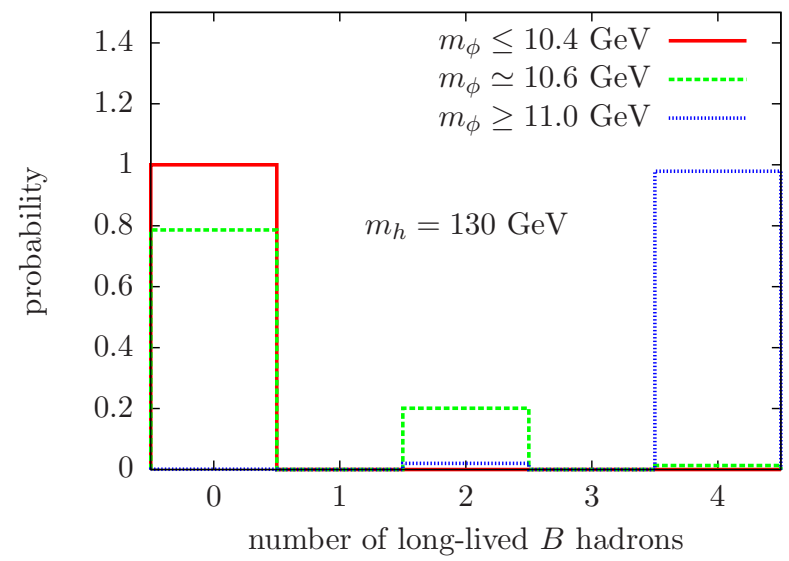

FIG. 6: We count the number of long lived $B$-hadrons with decay length $c \tau \geq 0.3 \mathrm{~mm}$ for a higgs decaying via $h \rightarrow \phi \phi \rightarrow$ $b \bar{b} b \bar{b}$.

hides the Higgs not only by decaying into jets but these jets are also softer. Examples of these scenarios are realized in Refs. [25, 58, 59]. Introducing cascades as in Eq. (2) produces more but softer radiation. Therefore to identify the Higgs decay products it is advantageous to produce the Higgs in a boosted state. Irrespective of the presence of a cascade this can help disentangle the Higgs from the background [59, 60]. This also helps when one uses a cascade ending in partially or completely leptonic final states which would otherwise be too soft to pass the cuts [61].

Another option is to decay the Higgs into gluons via a $\phi$ with a mass close to a heavy flavor bound state, e.g. $b \bar{b}$. In the $\phi$ rest frame the quarks are back to back and if their momenta are small it is likely for them to hadronize to a quark-anti quark bound state. Quarkanti quark bound states like $\Upsilon$ and $\eta_{b}$ typically decay dominantly into gluons. In this case no direct coupling of the $\phi$ to gluons and light quarks is necessary and this would be a generic feature if the $\phi$ couples Yukawa-like to quarks. One example is a cascade $h \rightarrow \phi \phi \rightarrow \Upsilon \Upsilon \rightarrow 6 g$. In Fig. 6] we use PYтhiA 8 [62] to simulate such a cascade. This example demonstrates that the $\phi$ needs to be quite close to threshold as already a relatively small mass difference between the $\phi$ and the quark-anti quark bound state significantly reduces the branching ratio to this bound state leaving us with a significant number of long lived B-hadrons. For other "heavy" quarks identification is more difficult to begin with as there are fewer clean long-lived states.

\section{Combined Higgs hide-outs}

The hide-outs outlined in the previous sections ЩA IIC can be combined and result in a large variety of signatures some of which can be even more challenging.

Let us imagine that the Higgs can decay via three different states denoted by $\phi_{\text {inv }}, \phi_{\text {disp }}$, and $\phi_{\text {jets }}$, where the subscript indicates the decay properties of the $\phi$ as invisible, long-lived or dominant decay into jets.

$h \rightarrow \phi_{\text {disp }}\left(\phi_{\text {inv }}, \phi_{\text {jets }}\right):$ Adapted trigger strategies to highly displaced vertices as outlined in [55] can obviously cope with signatures of these types as well, unless the majority of decays ocur outside the detector. Eventually reconstructing the mass of the Higgs boson is however more involved. This is due to the systematic uncertainties that enter in the measurement of the $\mathbb{E}_{T}$, which is sensitive to all energy deposited in the detector, or the jet energy scale.

$h \rightarrow \phi_{\text {inv }} \phi_{\text {jets }}$ : If the Higgs is produced at rest and $m_{h} \gg m_{\phi_{\text {jets }}}$ the resulting signature is a monojet with a missing energy $\sim m_{h} / 2$. In this case boosting the Higgs by recoiling it against a $Z$ or an additional jet is not necessarily a good strategy. Then the jet from $\phi_{\text {jets }}$ and the missing energy from $\phi_{\text {inv }}$ are aligned. This is problematic since it is already difficult to precisely measure the jet energy. Therefore it is difficult to measure missing energy aligned with jets. In fact, ATLAS and CMS typically apply cuts on extra jets in $\mathbb{E}_{T}$ searches to remove backgrounds, reducing the sensitivity toward these final states 63 . $Z+$ jets is already a non-negligible background if the Higgs recoils against a $Z$ but this problem becomes even more severe if the Higgs recoils against a jet.

\section{SUMMARY}

In this paper we have discussed phenomenological modifications of the Higgs sector which can serve to hide a Higgs and weaken the currently existing bounds. The Standard Model-like Higgs is a minimal solution to electroweak symmetry breaking. Therefore we focus on scenarios where the electroweak symmetry breaking sector is as in the Standard Model (SM). This limits the number of potential hide-outs for the Higgs boson and allows to classify them in terms of phenomenological signatures at colliders. We also suggest a simple benchmark model that parameterizes these possible signatures. Modified search strategies, looking at different signatures but also using adapted trigger strategies and elaborate reconstruction techniques (e.g. subjets) can help to uncover even an evasive Higgs.

There are essentially three possible hide-outs. One is to have dominantly invisible decays. This typically produces missing energy signatures. In this case a promising search channel is a monojet plus missing energy. We have used existing ATLAs data for this channel and constrained the production cross section times invisible branching fraction. For comparison we also estimated the sensitivity for searches using associated production.

A second hide out is for the Higgs is to first decay into long-lived neutral particles. Their eventual decay may then be observed in displaced vertex searches. Depending on the decay length it may be advantageous to also 
search for such decays occurring in the outer parts of the detector. This feature could even be enhanced by cascade decays.

Finally, the Higgs could decay dominantly to light quarks and gluons and be buried in huge QCD background. These decays can occur via cascades potentially including also intermediate $\bar{q} q$ bound states. Here, methods looking into specific radiation patterns in the jet substructure can be important to uncover the Higgs.

Of course, combining the different hide-outs can make searches even more difficult.

In general we find that hiding heavy Higgses is rather challenging from a theoretical point of view while light Higgses (for which $h \rightarrow V V$ is inaccessible) is more straightforward.

Current analysis are not yet sensitive enough to pin down all Higgs hide-outs. However, the ever increasing LHC data set together with a combined analysis of all sensitive channels will significantly tighten the con- straints for all hide-outs in the near future.

\section{Acknowledgements}

We thank Kristov Hackstein, Marc Hohlfeld, Peter Loch, Andrew Pilkington and David Strom for helpful discussions. CE acknowledges funding by the Durham International Junior Research Fellowship scheme. ER acknowledges financial support from the LHCPhenoNet network under the Grant Agreement PITN-GA-2010264564 .

The simulations underlying this study have been performed in parts on bwGRiD, member of the German D-Grid initiative, funded by the Bundesministerium für Bildung und Forschung and the Ministerium für Wissenschaft, Forschung und Kunst Baden-Württemberg.
[1] G. Aad et al. [Atlas Collaboration], JINST 3 (2008) S08003. G. L. Bayatian et al. [CMS Collaboration], J. Phys. G 34 (2007) 995.

[2] The Atlas Collaboration, arXiv:1112.2577 [hep-ex] ATLAS-CONF-2011-161, ATLAS-CONF-2011-162, ATLAS-CONF-2011-163.

[3] The CMS Collaboration, CMS-PAS-HIG-11-025, CMSPAS-HIG-11-030, CMS-PAS-HIG-11-031, CMS-PASHIG-11-032.

[4] R. Barate et al., Phys. Lett. B 565 (2003) 61.

[5] The Atlas collaboration, ATL-PHYS-PUB-2011-001.

[6] The Atlas collaboration, ATLAS-CONF-2011-134, ATLAS-CONF-2011-111.

[7] The CMS collaboration, CMS-HIG-11-014, CMS-PASHIG-11-003.

[8] The Atlas collaboration, ATLAS-CONF-2011-157. The CMS collaboration, CMS PAS HIG-11-023.

[9] The ALEPH, CDF, D0, DELPHI, L3, OPAL, SLD Collaborations, the LEP Electroweak Working Group, the Tevatron Electroweak Working Group, and the SLD electroweak and heavy flavour groups, arXiv:1012.2367 [hep$\mathrm{ex}]$.

[10] J. M. Cornwall, D. N. Levin and G. Tiktopoulos, Phys. Rev. Lett. 30 (1973) 1268 [Erratum-ibid. 31 (1973) 572]. J. M. Cornwall, D. N. Levin and G. Tiktopoulos, Phys. Rev. D 10 (1974) 1145 [Erratum-ibid. D 11 (1975) 972].

[11] K. Hagiwara, R. D. Peccei, D. Zeppenfeld and K. Hikasa, Nucl. Phys. B 282, 253 (1987).

[12] A. Bredenstein, K. Hagiwara and B. Jager, Phys. Rev. D 77 (2008) 073004.

[13] M. Ciccolini, A. Denner, S. Dittmaier, Phys. Rev. Lett. 99 (2007) 161803. M. Ciccolini, A. Denner and S. Dittmaier, Phys. Rev. D 77 (2008) 013002.

[14] J. Bagger et al., Phys. Rev. D 52 (1995) 3878. B. Jager, C. Oleari and D. Zeppenfeld, Phys. Rev. D 73 (2006) 113006. B. Jager, C. Oleari and D. Zeppenfeld, JHEP 0607 (2006) 015.

[15] U. Ellwanger, M. Rausch de Traubenberg and C. A. Savoy, Nucl. Phys. B 492 (1997) 21. R. Der- misek and J. F. Gunion, Phys. Rev. Lett. 95 (2005) 041801. U. Ellwanger, J. F. Gunion and C. Hugonie, JHEP 0507 (2005) 041. G. G. Ross and K. SchmidtHoberg, arXiv:1108.1284 [hep-ph].

[16] B. Gripaios, A. Pomarol, F. Riva and J. Serra, JHEP 0904 (2009) 070.

[17] M. J. Strassler and K. M. Zurek, Phys. Lett. B 651, 374 (2007).

[18] R. Schabinger and J. D. Wells, Phys. Rev. D 72 (2005) 093007. R. Foot, H. Lew and R. R. Volkas, Phys. Lett. B 272 (1991) 67. Z. Chacko, H. S. Goh and R. Harnik, Phys. Rev. Lett. 96 (2006) 231802. B. Patt and F. Wilczek, arXiv:hep-ph/0605188. V. Barger, P. Langacker, M. McCaskey, M. J. Ramsey-Musolf and G. Shaughnessy, Phys. Rev. D 77, 035005 (2008). T. Binoth and J. J. van der Bij, Z. Phys. C 75, 17 (1997). O. Bahat-Treidel, Y. Grossman and Y. Rozen, JHEP 0705, 022 (2007). A. Dedes, T. Figy, S. Hoche, F. Krauss and T. E. J. Underwood, JHEP 0811 (2008) 036.

[19] M. E. Peskin and T. Takeuchi, Phys. Rev. D 46, 381 (1992).

[20] R. Barbieri, A. Pomarol, R. Rattazzi and A. Strumia, Nucl. Phys. B 703, 127 (2004). G. F. Giudice, C. Grojean, A. Pomarol and R. Rattazzi, JHEP 0706 (2007) 045. K. Agashe and R. Contino, Nucl. Phys. B 742 (2006) 59. S. Bock, R. Lafaye, T. Plehn, M. Rauch, D. Zerwas and P. M. Zerwas, Phys. Lett. B 694 (2010) 44.

[21] C. Englert, T. Plehn, D. Zerwas and P. M. Zerwas, Phys. Lett. B 703 (2011) 298.

[22] K. Hagiwara, R. Szalapski and D. Zeppenfeld, Phys. Lett. B 318 (1993) 155. V. Hankele, G. Klamke, D. Zeppenfeld and T. Figy, Phys. Rev. D 74 (2006) 095001. F. Bonnet, M. B. Gavela, T. Ota and W. Winter, arXiv:1105.5140 [hep-ph].

[23] W. D. Goldberger, B. Grinstein and W. Skiba, Phys. Rev. Lett. 100 (2008) 111802 J. Fan, W. D. Goldberger, A. Ross and W. Skiba, Phys. Rev. D 79 (2009) 035017

[24] Y. Mambrini, arXiv:1108.0671 [hep-ph]. M. Raidal, 
A. Strumia, arXiv:1108.4903 [hep-ph]. R. Foot, A. Kobakhidze and R. R. Volkas, arXiv:1109.0919 [hepph]. X. -G. He, J. Tandean, Phys. Rev. D84, 075018 (2011). M. Pospelov, A. Ritz, arXiv:1109.4872 [hep-ph]. I. Low, P. Schwaller, G. Shaughnessy, C. E. M. Wagner, arXiv:1110.4405 [hep-ph]. E. Weihs and J. Zurita, arXiv:1110.5909 [hep-ph]. J. W. Cui, H. J. He, L. C. Lv and F. R. Yin, arXiv:1110.6893 [hep-ph]. S. V. Demidov, D. S. Gorbunov and A. A. Tokareva, arXiv:1111.1072 [hep-ph].

[25] B. Bellazzini, C. Csaki, A. Falkowski, A. Weiler, Phys. Rev. D81 (2010) 075017. B. Bellazzini, C. Csaki, A. Falkowski, A. Weiler, Phys. Rev. D80 (2009) 075008.

[26] D. Alves et al., arXiv:1105.2838 [hep-ph].

[27] L. B. Okun, Sov. Phys. JETP 56 (1982) 502 [Zh. Eksp. Teor. Fiz. 83 (1982) 892]. B. Holdom, Phys. Lett. B 166, 196 (1986).

[28] J. Jaeckel and A. Ringwald, Ann. Rev. Nucl. Part. Sci. 60 (2010) 405.

[29] S. Dodelson and L. M. Widrow, Phys. Rev. Lett. 72 (1994) 17 J. March-Russell, S. M. West, D. Cumberbatch and D. Hooper, JHEP 0807, 058 (2008).

[30] C. D. Froggatt, H. B. Nielsen, Nucl. Phys. B147 (1979) 277.

[31] I. Low, R. Rattazzi, A. Vichi, JHEP 1004 (2010) 126.

[32] V. Barger, P. Langacker, G. Shaughnessy, Phys. Rev. D75 (2007) 055013. V. Barger, P. Langacker, M. McCaskey, M. J. Ramsey-Musolf, G. Shaughnessy, Phys. Rev. D77 (2008) 035005. V. Barger, P. Langacker, M. McCaskey, M. Ramsey-Musolf, G. Shaughnessy, Phys. Rev. D79 (2009) 015018. V. Barger, H. E. Logan, G. Shaughnessy, Phys. Rev. D79 (2009) 115018.

[33] A. De Roeck et al., Eur. Phys. J. C 66 (2010) 525.

[34] K. Belotsky, V. A. Khoze, A. D. Martin and M. G. Ryskin, Eur. Phys. J. C 36 (2004) 503.

[35] LEP Higgs Working for Higgs boson searches, arXiv:hep-ex/0107032.

[36] A. Birkedal, K. Matchev, M. Perelstein, Phys. Rev. D70, 077701 (2004). J. L. Feng, S. Su and F. Takayama, Phys. Rev. Lett. 96, 151802 (2006) M. Beltran, D. Hooper, E. W. Kolb and Z. C. Krusberg, Phys. Rev. D 80, 043509 (2009) Q. -H. Cao, C. -R. Chen, C. S. Li, H. Zhang, JHEP 1108, 018 (2011). M. Beltran, D. Hooper, E. W. Kolb, Z. A. C. Krusberg, T. M. P. Tait, JHEP 1009, 037 (2010). J. Goodman, M. Ibe, A. Rajaraman, W. Shepherd, T. M. P. Tait, H. -B. Yu, Phys. Lett. B695, 185188 (2011). Y. Bai, P. J. Fox, R. Harnik, JHEP 1012, 048 (2010). P. J. Fox, R. Harnik, J. Kopp, Y. Tsai, Phys. Rev. D84, 014028 (2011). A. Rajaraman, W. Shepherd, T. M. P. Tait, A. M. Wijangco, arXiv:1108.1196 [hep-ph]. P. J. Fox, R. Harnik, J. Kopp, Y. Tsai, arXiv:1109.4398 [hep-ph].

[37] The Atlas collaboration, arXiv:1106.5327 [hep-ex], and ATLAS-CONF-2011-096 for the updated analysis of the $\mathcal{L}=1 \mathrm{fb}^{-1}$ data set.

[38] S. Hoeche, F. Krauss, M. Schonherr and F. Siegert, arXiv:1111.1220 [hep-ph].

[39] P. Nason, JHEP 0411, 040 (2004). S. Alioli, P. Nason, C. Oleari and E. Re, JHEP 0904, 002 (2009). S. Alioli, P. Nason, C. Oleari, E. Re, JHEP 1006, 043 (2010).

[40] T. Sjostrand, S. Mrenna, P. Z. Skands, JHEP 0605, 026 (2006).

[41] M. Cacciari, G. P. Salam, G. Soyez, JHEP 0804, 063 (2008). M. Cacciari, G.P. Salam and G. Soyez, http://fastjet.fr/

[42] A. L. Read, CERN-OPEN-2000-205. A. L. Read, J. Phys. G G28 (2002) 2693-2704.

[43] T. Junk, Nucl. Instrum. Meth. A 434 (1999) 435. T. Junk, CDF Note 8128 [cdf/doc/statistics/public/8128]. T. Junk, CDF Note 7904 [cdf/doc/statistics/public/7904]. H. Hu and J. Nielsen, in 1st Workshop on Confidence Limits, CERN 2000-005 (2000)

[44] T. Gleisberg, S. Hoeche, F. Krauss, M. Schonherr, S. Schumann, F. Siegert and J. Winter, JHEP 0902 (2009) 007; S. Schumann, F. Krauss, JHEP 0803 (2008) 038; S. Hoeche, F. Krauss, S. Schumann, F. Siegert, JHEP 0905 (2009) 053.

[45] J. M. Campbell and R. K. Ellis, Phys. Rev. D 60, 113006 (1999). J. M. Campbell and R. K. Ellis, arXiv:1007.3492 [hep-ph]. http://mcfm.fnal.gov

[46] G. D. Kribs, T. Plehn, M. Spannowsky and T. M. P. Tait, Phys. Rev. D 76 (2007) 075016.

[47] B. Holdom, W. S. Hou, T. Hurth, M. L. Mangano, S. Sultansoy and G. Unel, PMC Phys. A 3 (2009) 4.

[48] X. Ruan and Z. Zhang, arXiv:1105.1634 [hep-ph].

[49] K. Belotsky, D. Fargion, M. Khlopov, R. Konoplich and K. Shibaev, Phys. Rev. D 68 (2003) 054027. S. A. Cetin, T. Cuhadar-Donszelmann, M. Sahin, S. Sultansoy and G. Unel, arXiv:1108.4071 [hep-ph]. L. M. Carpenter, arXiv:1110.4895 [hep-ph].

[50] R. M. Godbole, M. Guchait, K. Mazumdar, S. Moretti and D. P. Roy, Phys. Lett. B 571 (2003) 184. H. Davoudiasl, T. Han and H. E. Logan, Phys. Rev. D 71 (2005) 115007.

[51] O. J. P. Eboli, D. Zeppenfeld, Phys. Lett. B495, 147-154 (2000).

[52] K. Arnold et al., Comput. Phys. Commun. 180, 1661 (2009); K. Arnold et al., arXiv:1107.4038 [hep-ph].

[53] The CMS collaboration, CMS-PAS-HIG-11-029.

[54] M. J. Strassler, K. M. Zurek, Phys. Lett. B661 (2008) 263-267.

[55] G. Ciapetti, ATL-COM-PHYS-2008-155. A. Nisati, S. Petrarca, G. Salvini, ATL-MUON-97-205. S. Ambrosanio, B. Mele, S. Petrarca, G. Polesello, A. Rimoldi, ATL-PHYS-2002-006. S. Tarem et al., ATL-PHYS-PUB2005.022. J. Ellis, A.R. Raklev, O.K. Oye, ATL-PHYSPUB-2007-016. S. Tarem, S. Bressler, H. Nomoto, A. Di Mattia, ATL-PHYS-PUB-2008-01.

[56] S. Dimopoulos, M. Dine, S. Raby and S. D. Thomas, Phys. Rev. Lett. 76 (1996) 3494. C. H. Chen and J. F. Gunion, Phys. Rev. D 58, 075005 (1998).

[57] Peter Loch, private communication.

[58] S. Chang, R. Dermisek, J. F. Gunion and N. Weiner, Ann. Rev. Nucl. Part. Sci. 58 (2008) 75.

[59] A. Falkowski, D. Krohn, L. -T. Wang, J. Shelton, A. Thalapillil, arXiv:1006.1650 [hep-ph]. C. R. Chen, M. M. Nojiri and W. Sreethawong, JHEP 1011 (2010) 012. B. Bellazzini, C. Csaki, J. Hubisz and J. Shao, Phys. Rev. D 83 (2011) 095018 C. Englert, T. S. Roy and M. Spannowsky, arXiv:1106.4545 [hep-ph].

[60] J. M. Butterworth, A. R. Davison, M. Rubin, G. P. Salam, Phys. Rev. Lett. 100, 242001 (2008). T. Plehn, G. P. Salam, M. Spannowsky, Phys. Rev. Lett. 104, 111801 (2010). G. D. Kribs, A. Martin, T. S. Roy and M. Spannowsky, Phys. Rev. D 81 (2010) 111501. D. E. Soper, M. Spannowsky, JHEP 1008, 029 (2010). L. G. Almeida, S. J. Lee, G. Perez, G. Sterman, I. Sung, 
Phys. Rev. D82, 054034 (2010). G. D. Kribs, A. Martin, T. S. Roy, M. Spannowsky, Phys. Rev. D82, 095012 (2010). C. Hackstein, M. Spannowsky, Phys. Rev. D82, 113012 (2010). C. Englert, C. Hackstein, M. Spannowsky, Phys. Rev. D82, 114024 (2010). D. E. Soper, M. Spannowsky, Phys. Rev. D84, 074002 (2011). J. -H. Kim, Phys. Rev. D83, 011502 (2011). J. Gallicchio, J. Huth, M. Kagan, M. D. Schwartz, K. Black, B. Tweedie, JHEP 1104, 069 (2011). J. Thaler, K. Van Tilburg, JHEP 1103, 015 (2011). A. Katz, M. Son, B. Tweedie, Phys. Rev. D83, 114033 (2011). J. Thaler, Z. Thomas, JHEP
1107, 060 (2011)

[61] C. Cheung, J. T. Ruderman, L. T. Wang and I. Yavin, JHEP 1004 (2010) 116. A. Falkowski, J. T. Ruderman, T. Volansky and J. Zupan, JHEP 1005 (2010) 077; Phys. Rev. Lett. 105 (2010) 241801.

[62] T. Sjostrand, S. Mrenna, P. Z. Skands, Comput. Phys. Commun. 178, 852-867 (2008).

[63] The Atlas Collaboration, Phys. Rev. Lett. 106, 131802 (2011). The CMS Collaboration, Phys. Lett. B 698 (2011) 196. 